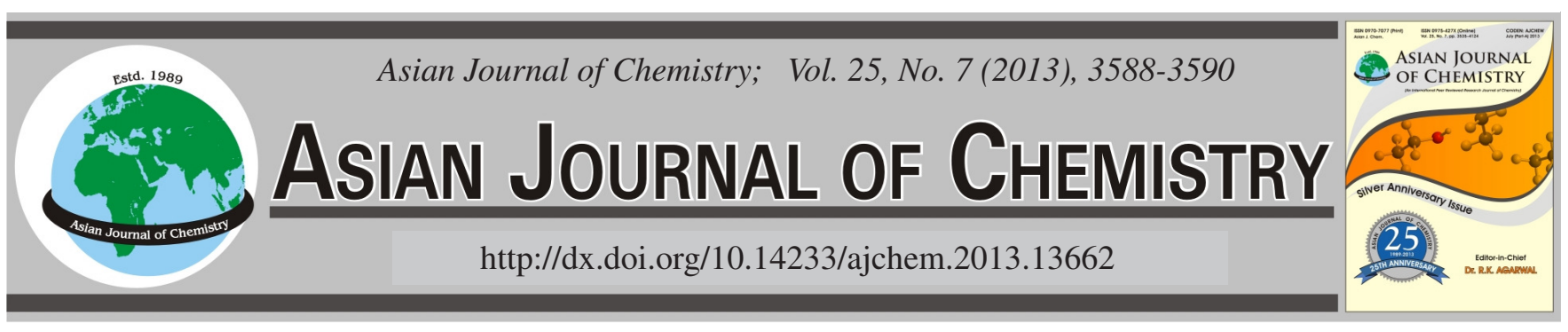

\title{
An Efficient Method for the Synthesis of Isoxazolines Under Microwave Irradiation and Solvent-Free Conditions
}

\author{
Pravin O. PatiL ${ }^{*}$ and SAnJay. B. Bari
}

Department of Pharmaceutical Chemistry, R.C. Patel Institute of Pharmaceutical Education and Research, Shirpur-425 405, India

*Corresponding author: E-mail: rxpatilpravin@yahoo.co.in

(Received: 28 February 2012;

Accepted: 7 January 2013)

AJC-12654

\begin{abstract}
A facile method for the synthesis of new isoxazoline derivatives are being reported starting from substituted 1-(1H-indol-3-yl)-3-(substituted aryl)-prop-1-en-3-ones and hydroxylamine hydrochloride under solvent free conditions and microwave irradiation. This approach has advantages such as short reaction time, moderate to excellent yield, over the conventional synthesis. The structures of the compounds were confirmed by ${ }^{1} \mathrm{H}$ NMR, IR, mass spectra and elemental $(\mathrm{C}, \mathrm{H}, \mathrm{N})$ analysis.

Key Words: Solid phase synthesis, Isoxazolines, Indole, Microwave assisted synthesis.

- - - - - - - - - - - - - - - - - - - - - - - - - - -
\end{abstract}

\section{INTRODUCTION}

Indole nucleus is the most widely used heterocyclic ring for the development of bioactive molecules. Indole derivatives have occupied unique place in medicinal and biological chemistry due to their diverse pharmacological activities ${ }^{1-3}$. Recent literature is enriched with progressive findings about the synthesis and pharmacological potentials of various indole and fused indole derivatives ${ }^{4-6}$.

Compounds containing isoxazole nucleus have been reported to possess anticancer, antimicrobial, antinociceptive, macrophage migration inhibitory factor activity, antimicrobial, antioxidant, protein tyrosine phosphatases 1B inhibitors, antipsychotic, anticonvulsant, antidepressant agents ${ }^{7-13}$. Because of their wide range of biological, industrial and synthetic applications, fused indole derivatives as well as isoxazolines have recently received a great deal of attention. In view of these observations, we aimed to synthesize novel derivatives of indole fused with isoxazoline nucleus with enhanced pharmacological potentials.

From the literature survey, it was found that large numbers of isoxazoline derivatives of pharmacological significance were synthesized by the action of hydroxylamine hydrochloride on chalcones in presence of base ${ }^{14-19}$. In present communication we have synthesized isoxazolines from chalcones and hydroxylamine hydrochloride using basic alumina as solid support under microwave and the structures of the compounds were confirmed by ${ }^{1} \mathrm{H}$ NMR, IR, mass spectra and elemental $(\mathrm{C}, \mathrm{H}$, $\mathrm{N}$ ) analysis. A conventional method has several limitations such as long reaction time, use of harmful reagents like acetic acid hence we have reported green approach for the synthesis of isoxazolines.

During the last decade, the topic of solid phase microwave assisted synthesis has received increasing attention. Microwave-assisted reactions in solvents have several limitations like the development of high pressures, need for specialized reaction vessels and superheating of solvents leads to solvent loss during reaction are circumvented via solid support technique which enables organic reactions to occur rapidly at atmospheric pressure. While the overwhelming majority of organic reactions require several hours to achieve a substantial product yield, the analogous reactions in the solid phase occur practically within minute ${ }^{20,21}$. Therefore, we have reported here a microwave-accelerated solid state approach for the rapid synthesis of 5-(1H-indol-3-yl)-3-(4-substituted aryl)-4, 5-dihydroisoxazoline derivatives.

In the present work, the isoxazolines, $\left(\mathrm{II}_{\mathrm{A}}-\mathrm{II}_{\mathrm{H}}\right)$, were synthesized by basic alumina supported solid phase cyclocondensation of 1- (1H-indol-3-yl)-3-(substituted aryl)-prop1-en-3-one in the presence of hydroxylamine hydrochloride under microwave utilizing the reaction sequence as shown in Scheme-I.

\section{EXPERIMENTAL}

Title compounds indolyl isoxazolines were synthesized by literature method ${ }^{22}$. Melting points were measured and uncorrected on Elico melting point apparatus using open capillary tube. All microwave assisted solid phase reactions 
were performed using a scientific microwave oven (catalyst electromagnetic System) with a power of $800 \mathrm{~W}$ specially designed for organic synthesis. Thin-layer chromatography was performed on precoated plates $\mathrm{SiO}_{2}$ (silica gel 60, F 254, Merck) to monitor progress of the reaction and purity of the compounds. Silica gel [60-120 mesh size), Merck] was used for column chromatography. IR spectra of compounds were recorded on Schimadzu IR 48 Spectrophotometer. All the NMR spectra were recorded on Bruker Avance III, $400 \mathrm{MHz}$ spectrometer with $\mathrm{CDCl}_{3} / \mathrm{DMSO}$ as solvent. Mass spectra were recorded on 410 Prostar Binary LC with 500 MS IT PDA detectors.

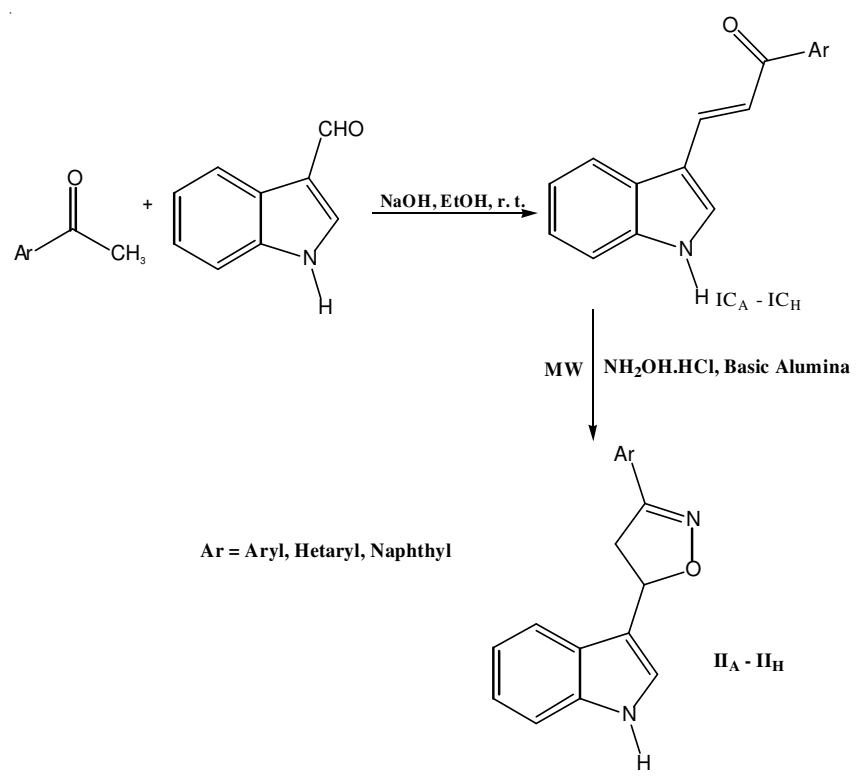

Scheme-I: Synthetic route for the synthesis of target compounds $\left(\mathrm{II}_{\mathrm{A}}-\mathrm{II}_{\mathrm{H}}\right)$

\begin{tabular}{|c|c|c|c|}
\hline \multicolumn{4}{|c|}{$\begin{array}{c}\text { TABLE-1 } \\
\text { PHYSICO-CHEMICAL PROPERTIES OF SYNTHESIZED } \\
\text { ISOXAZOLINES VIA SCHEME-I }\end{array}$} \\
\hline Compound & $\mathrm{Ar}$ & Yield $(\%)^{\mathrm{a}}$ & m.p. $\left({ }^{\circ} \mathrm{C}\right)$ [L. m.p.] \\
\hline $\mathrm{II}_{\mathrm{A}}$ & Phenyl & 89.92 & $160-162$ \\
\hline $\mathrm{II}_{\mathrm{B}}$ & 4-Chlorophenyl & 84.34 & $176-177$ \\
\hline $\mathrm{II}_{\mathrm{C}}$ & 4-Methylphenyl & 83.57 & $165-166[167]^{25}$ \\
\hline $\mathrm{II}_{\mathrm{D}}$ & 2-Pyridyl & 78.82 & 196-197 \\
\hline $\mathrm{II}_{\mathrm{E}}$ & 4-Fluorophenyl & 74.62 & 205-207 \\
\hline $\mathrm{II}_{\mathrm{F}}$ & 4-Methoxyphenyl & 81.27 & $170-173$ \\
\hline $\mathrm{II}_{\mathrm{G}}$ & 2-Naphthyl & 80.24 & 204-206 \\
\hline $\mathrm{II}_{\mathrm{H}}$ & 4-Nitrophenyl & 86.62 & $192-195$ \\
\hline
\end{tabular}

Synthesis of 5-(1H-Indol-3-yl)-3-(substituted aryl)-4, 5-dihydroisoxazoline derivatives $\left(\mathbf{I I}_{\mathrm{A}}-\mathbf{I I}_{\mathrm{H}}\right)$ : A mixture of 1-(1H-indol-3-yl)-3-(substituted aryl)-prop-1-en-3-ones $(1 \mathrm{mmol})$ and hydroxylamine hydrochloride $(2 \mathrm{mmol})$ in $\mathrm{EtOH}$ was added basic alumina $(20 \mathrm{~g})$ in $100 \mathrm{~mL}$ beaker. The reaction mixture was thoroughly stirred and dried in air. The reactants loaded on basic alumina were placed in an alumina bath ${ }^{23}$ and were irradiated in the scientific microwave oven intermittently at $30 \mathrm{~s}$ interval for specified time at 455 watt. Reaction progress was monitored by TLC. Reaction mixture was cooled to room temperature after completion of reaction and the product was extracted into organic solvent and was kept in air after partial evaporation of solvent fine crystals of isoxazolines were formed. The resulting products were purified by column chromatography (chloroform/acetone 16:1 as eluant) to gave a desired products $\left(\mathrm{II}_{\mathrm{A}}-\mathrm{II}_{\mathrm{H}}\right)$. Melting point, IR and ${ }^{1} \mathrm{H}$ NMR spectra of compound $\mathrm{II}_{\mathrm{C}}$ was found to be identical with the one described in literature ${ }^{24}$ (Table-1)

5-(1H-Indol-3-yl)-3-(phenyl)-4,5-dihydroisoxazoline $\left(\mathbf{I I}_{\mathrm{A}}, \mathbf{C}_{\mathbf{1 7}} \mathbf{H}_{\mathbf{1 4}} \mathbf{N}_{\mathbf{2}} \mathbf{O}\right)$ : Yield: $89.92 \%$; m.p.: $160-162^{\circ} \mathrm{C}$; reaction time: $11 \mathrm{~min} ;{ }^{1} \mathrm{H}$ NMR (400 MHz, $\mathrm{CDCl}_{3}$ ) 6.02-6.07 (dd, $\left.1 \mathrm{H}\right)$, 3.55-3.62 (dd, 1H), 3.69-3.70 (dd, 1H), 7.09-7.76 (m, 10H), 8.175 (brs, 1H) ppm; IR (KBr, $\left.v_{\max }, \mathrm{cm}^{-1}\right): 3335(\mathrm{~N}-\mathrm{H}), 1615$ $(\mathrm{C}=\mathrm{C}), 1572(\mathrm{C}=\mathrm{N})$; MS: $m / z$ with +ve mode: $263.2\left(\mathrm{M}^{+}\right)$, $264.3(\mathrm{M}+1)$.

5-(1H-indol-3-yl)-3-(4-chlorophenyl)-4,5-dihydroisoxazoline $\left(\mathbf{I I}_{\mathbf{B}}, \mathbf{C}_{17} \mathbf{H}_{13} \mathbf{N}_{\mathbf{2}} \mathbf{O C l}\right)$ : Yield: $84.34 \%$; m.p.: 176$177{ }^{\circ} \mathrm{C}$; reaction time: $8 \mathrm{~min} ;{ }^{1} \mathrm{H}$ NMR $\left(400 \mathrm{MHz}, \mathrm{CDCl}_{3}\right.$ ) 6.03-6.08 (dd, 1H), 3.27-3.59 (dd, 1H), 3.52-3.59 (dd, 1H), 7.10-7.68 (m, 9H), 8.15 (brs, 1H) ppm; IR (KBr, $\left.v_{\max }, \mathrm{cm}^{-1}\right)$ : $3410(\mathrm{~N}-\mathrm{H}), 1616(\mathrm{C}=\mathrm{C}), 1588(\mathrm{C}=\mathrm{N})$; MS: $m / z$ with +ve mode: $297.1\left(\mathrm{M}^{+}\right), 298.2(\mathrm{M}+1)$.

5-(1H-Indol-3-yl)-3-(4-methylphenyl)-4,5-dihydroisoxazoline $\left(\mathbf{I I}_{\mathrm{C}}, \mathbf{C}_{\mathbf{1 8}} \mathbf{H}_{\mathbf{1 6}} \mathbf{N}_{\mathbf{2}} \mathbf{O}\right)$ : Yield: $83.27 \%$; m.p.: $165-166^{\circ} \mathrm{C}$; reaction time:- $10 \mathrm{~min} ;{ }^{1} \mathrm{H} \mathrm{NMR}\left(400 \mathrm{MHz}, \mathrm{CDCl}_{3}\right) 2.39$ (s, $3 \mathrm{H})$, 5.99-6.04 (dd, 1H), 3.53-3.60 (dd, 1H), 3.68-3.74 (dd, 1H), 7.09-7.64 (m, 9H), 8.15 (brs, 1H) ppm; IR (KBr, $v_{\max }$, $\left.\mathrm{cm}^{-1}\right)$ : $3424(\mathrm{~N}-\mathrm{H}), 1610(\mathrm{C}=\mathrm{C}), 1635(\mathrm{C}=\mathrm{N})$; MS: $\mathrm{m} / z$ with +ve mode: $277.2\left(\mathrm{M}^{+}\right), 278.1(\mathrm{M}+1)$.

5-(1H-indol-3-yl)-3-(2-pyridyl)-4,5-dihydroisoxazoline $\left(\mathbf{I I}_{\mathrm{D}}, \mathbf{C}_{\mathbf{1 6}} \mathbf{H}_{\mathbf{1 3}} \mathbf{N}_{\mathbf{3}} \mathbf{O}\right)$ : Yield: $78.82 \%$; m.p.: $196-197^{\circ} \mathrm{C}$; reaction time: $14 \mathrm{~min} ;{ }^{1} \mathrm{H}$ NMR $\left(400 \mathrm{MHz}, \mathrm{CDCl}_{3}\right) 6.01-6.98(\mathrm{dd}, 1 \mathrm{H})$, 3.63-3.69 (dd, 1H), 3.79-3.87 (dd, 1H), 6.98-8.94 (m, 9H), 11.17 (brs, 1H) ppm; IR (KBr, $\left.v_{\max }, \mathrm{cm}^{-1}\right): 3210(\mathrm{~N}-\mathrm{H}), 1594$ $(\mathrm{C}=\mathrm{C}), 1616(\mathrm{C}=\mathrm{N})$; MS: $m / z$ with +ve mode: $265.12\left(\mathrm{M}^{+}\right)$, $266.2(\mathrm{M}+1)$.

5-(1H-indol-3-yl)-3-(4-fluorophenyl)-4,5-dihydroisoxazoline ( $\left.\mathbf{I I}_{\mathrm{E}}, \mathbf{C}_{18} \mathbf{H}_{\mathbf{1 7}} \mathbf{N}_{\mathbf{2}} \mathbf{O F}\right)$ : Yield: $74.62 \%$; m.p.: 205-207 ${ }^{\circ} \mathrm{C}$; reaction time: $14 \mathrm{~min} ;{ }^{1} \mathrm{H}$ NMR $\left(400 \mathrm{MHz}, \mathrm{CDCl}_{3}\right) 6.03$ 6.05 (dd, 1H), 3.53-3.59 (dd, 1H), 3.67-3.74 (dd, 1H), 6.097.67 (m, 9H), 8.154 (brs, 1H) ppm; IR (KBr, $\left.v_{\max }, \mathrm{cm}^{-1}\right): 3256$ $(\mathrm{N}-\mathrm{H}), 1608(\mathrm{C}=\mathrm{C}), 1583(\mathrm{C}=\mathrm{N})$; MS: $\mathrm{m} / \mathrm{z}$ with +ve mode: $297.32\left(\mathrm{M}^{+}\right), 298.21(\mathrm{M}+1)$.

5-(1H-indol-3-yl)-3-(4-methoxyphenyl)-4,5-dihydroisoxazoline $\left(\mathrm{II}_{\mathrm{F}}, \mathbf{C}_{18} \mathbf{H}_{16} \mathbf{N}_{2} \mathbf{O}_{2}\right)$ : Yield: $84.57 \%$; m.p.: 170$173{ }^{\circ} \mathrm{C}$; reaction time: $15 \mathrm{~min}$; ${ }^{1} \mathrm{H}$ NMR $\left(400 \mathrm{MHz}, \mathrm{CDCl}_{3}\right.$ ) 3.79 (s, 3H), 5.92-6.01 (dd, 1H), 3.59-3.61 (dd, 1H), 3.633.78 (dd, 1H), 7.11-7.61 (m, 9H), 8.16 (brs, 1H) ppm; IR (KBr, $\left.v_{\max }, \mathrm{cm}^{-1}\right): 3251(\mathrm{~N}-\mathrm{H}), 1607(\mathrm{C}=\mathrm{C}), 1612(\mathrm{C}=\mathrm{N})$; MS: $\mathrm{m} / \mathrm{z}$ with +ve mode: $293.3\left(\mathrm{M}^{+}\right)$.

5-(1H-indol-3-yl)-3-(2-naphthyl)-4,5-dihydroisoxazoline $\left(\mathbf{I I}_{\mathbf{G}}, \mathbf{C}_{15} \mathbf{H}_{13} \mathbf{N}_{\mathbf{3}} \mathbf{O}\right)$ : Yield: $80.24 \%$; m.p.: 204-206 ${ }^{\circ} \mathrm{C}$; reaction time: $10 \mathrm{~min} ;{ }^{1} \mathrm{H}$ NMR $\left(400 \mathrm{MHz}, \mathrm{CDCl}_{3}\right) 6.02-$ 6.07 (dd, 1H), 3.55-3.62 (dd, 1H), 3.68-3.71 (dd, 1H), 7.02$7.78(\mathrm{~m}, 12 \mathrm{H}), 8.17$ (brs, $1 \mathrm{H}) \mathrm{ppm}$; IR $\left(\mathrm{KBr}, v_{\max }, \mathrm{cm}^{-1}\right): 3253$ $(\mathrm{N}-\mathrm{H}), 1618(\mathrm{C}=\mathrm{C}), 1582(\mathrm{C}=\mathrm{N})$; MS: $\mathrm{m} / \mathrm{z}$ with +ve mode: $313.21\left(\mathrm{M}^{+}\right)$.

5-(1H-indol-3-yl)-3-(4-nitrophenyl)-4,5-dihydroisoxazoline $\left(\mathbf{I I}_{\mathbf{H}}, \mathbf{C}_{17} \mathbf{H}_{13} \mathbf{N}_{3} \mathbf{O}_{3}\right)$ : Yield: $86.62 \%$; m.p.: 192-195 ${ }^{\circ} \mathrm{C}$; reaction time: $14 \mathrm{mins}$; ${ }^{1} \mathrm{H}$ NMR $\left(400 \mathrm{MHz}, \mathrm{CDCl}_{3}\right) 6.03$ $6.06(\mathrm{dd}, 1 \mathrm{H}), 3.52-3.59$ (dd, 1H), 3.67-3.74 (dd, 1H), 6.08- 
7.68 (m, 9H), 8.150 (brs, 1H) $3216(\mathrm{~N}-\mathrm{H}), 1621$ (C=C), 1576 $(\mathrm{C}=\mathrm{N})$; MS: $m / z$ with +ve mode: $308.2\left(\mathrm{M}^{+}\right), 309.14(\mathrm{M}+1)$.

\section{RESULTS AND DISCUSSION}

Based on the literature review, no reports were found on the synthesis of indolyl isoxazolines from indolyl chalcones by solid phase synthesis. We reports synthesis of 5-( $1 \mathrm{H}$-indol3-yl)-3-(4-substituted aryl)-4,5-dihydroisoxazoline derivatives using basic alumina without organic solvent under microwave irradiations. For our initial study we selected indolyl chalcones ( 1 equiv) and hydroxylamine hydrochloride (1 equiv) as model substrate using basic alumina as solid support and carried out reaction at 455 watt under microwave irradiations. We observed that, this system requires long reaction time (30-40 $\mathrm{min}$ ) to yield corresponding isoxazolines and the yield was poor hence we tried to determine the best chalcone to hydroxylamine hydrochloride ratio. With this aim we carried out the synthesis of isoxazolines $\mathrm{II}_{\mathrm{A}}-\mathrm{II}_{\mathrm{H}}$ using different equivalents of chalcones with hydroxylamine hydrochloride (ratio =1:1, 1:1.5, 1:2, 1:3). The yields obtained without purification after 20 minutes of reaction $(56,64,90$ and 72 respectively) show that chalcone in the ratio of $1 / 2$ to the hydroxylamine hydrochloride was the optimal composition hence we have synthesized all $\mathrm{II}_{\mathrm{A}}-\mathrm{II}_{\mathrm{H}}$ derivatives at the same optimized reaction conditions. The IR spectrum of the isoxazolines confirmed by formation of absorption bands of $\mathrm{C}=\mathrm{N}$ between $1625-1570 \mathrm{~cm}^{-1}, \mathrm{C}=\mathrm{C}$ between $1630-1590 \mathrm{~cm}^{-1}$ and further confirmed by disappearance of carbonyl functional of indolyl chalcones between $1700-1645 \mathrm{~cm}^{-1}$.

In summary, we have developed a practical and cost effective, fast, ecofriendly procedure for solid phase synthesis of 5-(1H-indol-3-yl)-3-(4-substitutedaryl)-4, 5-dihydroisoxazoline derivatives under microwave irradiations. The notable advantages offered by this protocol over the conventional syntheses are the reduced reaction times, high purity and better yields of the products makes this procedure eco-friendly for synthesis.

\section{ACKNOWLEDGEMENTS}

The authors are thankful to Dr. S.J. Surana, Principal of the institute and Dr. S.G. Gattani, Principal HRPIPER, Shirpur for providing research facilities to carryout the work. Authors gratefully acknowledge SAIF, IIT, Powai and STIC, Cochin University, Cochin for spectral data.

\section{REFERENCES}

1. R. Preeti, V.K. Srivastava and K. Ashok, Eur. J. Med. Chem., 39, 449 (2004).

2. N. Siddiqui and M.S.A. Ahsan, Acta Pharm., 58, 445 (2008).

3. J.S. Biradar, B.S. Sasidhar and R. Parveen, Eur. J. Med. Chem., 45, 4074 (2010).

4. C. Mengchao, O. Masahiro, K. Hiroyuki, L.L. Bo and S. Hideo, Bioorg. Med. Chem. Lett., 21, 980 (2011).

5. S.S. Panda and P.V.R. Chaudhari, Indian J. Pharm. Sci., 70, 208 (2008).

6. U. Misra, A. Hitkari, A.K. Saxena, S. Gurtu and S. Shanker, Eur. J. Med. Chem., 31, 629 (1996).

7. S.Y. Hae, J.L. Eun, E.L. Jie, K.P. Woo, J.B. Du, S.C. Yong, Y.K. Hun, C. Hyunah and N.P. Ae, Bull. Korean. Chem. Soc., 30, 1873 (2009).

8. A. Padmaja, T. Payani, G.D. Reddy and V. Padmavathi, Eur. J. Med. Chem., 44, 4557 (2009).

9. T. Karthikeyan, V. Seelan, K.G. Lalitha and P.T. Perumal, Bioorg. Med. Chem. Lett., 19, 3370 (2009).

10. K.J. Ahmed, S. Reddy, M.J. Ramaiah, D.E. Dastagiri, V.M. Bharathi, A.F. Ameruddin, S.N.C.V.L. Pushpavalli, M. Pal-Bhadra, A. Juvekar, S. Sen and S. Zingde, Eur. J. Med. Chem., 45, 3924 (2010).

11. Y.R. Prasad, P.R. Kumar and B. Ramesh, Int. J. Chem. Sci., 5, 542 (2007).

12. S. Balachandran, P.K. Gadekar, S. Parkale, V.N. Yadav, D. Kamath, S. Ramaswamy, S. Sharma, R.A. Vishwakarma and N.M. Dagia, Bioorg. Med. Chem. Lett., 21, 1508 (2011).

13. G. Ahmad, P. Mishra, K. Mishra, P. Gupta, P.P. Yadav, P. Tiwari, A.K. Tamarkar, A.K. Srivastava and R. Maurya, Bioorg. Med. Chem. Lett., 15, 2139 (2006).

14. A.A.R. Mohamed and M.H.A. Eman, Monatsh. Chem., 140, 229 (2009).

15. T. Shah and V. Desai, J. Serb. Chem. Soc., 72, 443 (2007).

16. S.B. Lohiya and B.J. Ghiya, Indian J. Chem., 25B, 279 (1996).

17. S.R. Mohane, V.G. Thakare and B.N. Berad, Asian J. Chem., 21, 7422 (2009).

18. V. Tiwari, P. Ali and J. Meshram, Ultrasonics Sonochem., 18, 911 (2011).

19. M. Kidwai, S. Kukreja and R. Thakur, Lett. Org. Chem., 3, 135 (2006).

20. S. Caddick, Tetrahedron, 51, 10403 (1995).

21. D. Dhabbi and C. Sharma, Indian J. Chem., 48B, 1006 (2009).

22. S.S. Panda, P.V.R. Chaudhari and B.S. Jayshree, Indian J. Pharm. Sci., 6845 (2009).

23. G. Bram, A. Loupy and M. Majdoub, Tetrahedron, 46, 5167 (1990).

24. X. Guo, X. Qi and J. Chang, Acta Cryst., E62, 0374, (2006). 